International Journal of Engineering \& Technology, $7(2.21)(2018) 161-165$
International Journal of Engineering \& Technology
Website: $w$ ww.sciencepubco.com/index.php/IJET
Research paper

\title{
Optimal Deployment of Camera Mounted UAVs Performing Search
}

\author{
Jeane Marina D'Souza ${ }^{1 *}$, Siddhartha Suresh Rao ${ }^{2}$, K.R. Guruprasad ${ }^{3}$ \\ ${ }^{1}$ Doctoral Student, Department of Mechanical Engineering, National Institute of Technology Karnataka \\ Surathkal, Mangaluru, India \\ ${ }^{2}$ Mechatronics Systems Engineer, Sasyaka Engineering, Bengaluru \\ ${ }^{3}$ Assistant Professor, Department of Mechanical Engineering, National Institute of Technology Karnataka, \\ Surathkal, Mangaluru, India \\ *Corresponding author E-mail:jeanemdsilva@gmail.com
}

\begin{abstract}
In this paper we address a problem of optimal deployment of camera mounted UAVs for a multi-robot search application. Here multiple UAVs carrying downward facing cameras are required to look for targets of interest in a search area. The lack of information about the presence or absence of targets is modeled as an uncertainty density distribution over the search area and this uncertainty is reduced as the information is gathered using the onboard cameras. The UAVs are required to get deployed so as to maximize the uncertainty reduction. We provide a model for search effectiveness of the camera and use it to formulate a strategy for optimal deployment of UAVs. It is shown that a centroidal Voronoi configuration, where each UAV (camera) is located at the centroid of the corresponding Voronoi cell is an optimal deployment. We provide simulation results to demonstrate that the proposed optimal deployment strategy successfully deploys the UAVs into centroidal Voronoi configuration, which maximizes the uncertainty reduction using cameras as search sensors.
\end{abstract}

Keywords: Camera As Search Sensor, Deployment, Multi-UAV search experiment, Uncertainty Reduction.

\section{Introduction}

The problem of searching for targets of interest using multiple aerial robots equipped with necessary search sensors has been addressed widely in the literature. Use of multiple searchers has several advantages such as reduced time of completion and robustness to failure of a few individual search agents. Major components of the multi-agent search problem are, path planning or deployment of the search agents, modeling the search process, and finally information extraction from the (search) sensory data. Though all components are interrelated, most work in the literature focus on one of these components, while assuming a suitable model/solution for other components. For example, authors such as in [4], [5] devise plan for the agent motion assuming that search effectiveness of a search sensor to be maximum directly below it and decreasing away from the center. In [6], authors present a multi-UAV search problem using cameras as search sensors. Authors address all components of the multi-agent search problem.

Camera's field of view (FOV) is used as the search sensor footprint, and whenever a point (center of a gridded region) comes under the FOV of any camera, then it is considered to be covered by the sensor. Effectiveness of the camera is assumed to be same at any point within its FOV, unlike in [4], [5]. In the context of distributed environmental monitoring, authors in [7] address a visual sensor coverage problem using multiple cameras mounted on UAVs. Though every point within a camera's FOV is considered covered, authors use the minimum information per pixel principle as a cost function for camera placement.
In this paper, we propose a search effectiveness model for a camera in line with that assumed in [4]. We formulate the problem of optimal deployment of UAVs carrying cameras, maximizing a suitably defined search effectiveness as the objective function. We show that a centroidal Voronoi configuration, where each camera is located at the centroid of the corresponding Voronoi cell computed based on the positions of the UAVs/cameras, solves the optimal deployment problem.

\section{The problem setting}

In this section we present the multi-UAV search strategy. The theoretical background is based on a search strategy presented in [4]. We consider a problem of multiple quadcopter covering an area of interest, or the search space, searching for presence of some targets of interest, using downward facing cameras.

The problem here is to devise a strategy for optimal deployment of multi-quadcopter so as to maximize the effectiveness of the search using on-board cameras in terms of information gain.

\section{Camera as a search sensor}

In most conditions, such as search and rescue operation in a natural calamity hit region, the targets such as survivors requiring immediate assistance or the amount of damage to be assessed, are typically visually detectable. A camera is suitable in most similar scenarios. 
UAVs such as quadcopters are usually equipped with a frontal camera and a downward facing camera. In most situations in an aerial search, downward facing cameras are more suitable. In a typical multi-UAV search problem, the cameras capture image of a large area. The search effectiveness function of a camera captures the spatial variation of effectiveness of the camera in terms of target detection capability. Successful target detection depends on the image quality. Most work on multi-agent search uses a flat effectiveness function. Such a sensor (search) effectiveness model is used in several including [6]. Such a model for camera may be suitable only for situations which provide clear and high contrast images. Either a high resolution camera is used or the targets are clearly distinguishable in the search area. This may not be true in many practical scenarios.

Search strategies presented in [4] use a model where a sensor is most effective at its centre, and the effectiveness monotonically reduces away from it. Cortes et al [8] also use a similar sensor effectiveness function for sensor coverage optimization problem. An exponential function is used in [4], for a generic search sensor. A camera has an optical system, a photo-sensor (such as CCD or CMOS), and signal processing systems. The original scene may get degraded in each of these stages. While some of the factors affect the image quality more or less uniformly throughout the image frame, some factors affect the image quality non-uniformly across the frame.

\subsection{Quantum efficiency: Camera Effectiveness}

Now we discuss the effectiveness of camera in a search scenario. The number of pixels and size of pixel are factors that affect the resolution of a camera. QE (Quantum Efficiency), is a measure of how efficiently the sensor converts light (photons) to charge (electrons). The more electrons in a pixel during the integration period, the higher the output level of the sensor, so the more sensitive the sensor is for that specific wavelength of the light [13]. By reducing the pixel size and increasing the number of pixels the resolution can be improved. But reducing the pixel size reduces the quantum efficiency (QE) of the sensor and increases the effect of vignetting. Vignetting, also known as "light fall-off" is common in optics and photography, which in simple terms means darkening of image corners when compared to the centre. Light travels through a narrow tunnel in going from the chip surface to a photo-detector in a CMOS sensor. This especially causes problems when light is incident at oblique angles since the narrow tunnel walls cast a shadow on the photodetector which will severely reduce its effective QE. Quantum efficiency (QE) is another metric that provides us a measure of effectiveness of the camera. While Modulation Transfer function (MTF) describes the spatial variation of light as a function of spatial frequency, QE describes the ratio of the total photons incident on the system to the number of photons producing charge carriers. Note that MTF is related to the optical system, while QE is related to the photodetective sensor. Chen [11] has presented some experimental results showing the variation in quantum efficiency of a camera with a CMOS sensor at various distances from the central pixel using simulation software. We have used these results and are plotted the curve on MATLAB. Figure 1 shows QE v/s distance from central pixel for a $6 \mathrm{~m}$ pixel size CMOS sensor from which we have obtained the following expression:

$$
f=0.8708-0.0714 \times r_{p i x}{ }^{2}
$$

Here, $r_{\text {pix }}$ is the distance of the pixel in question from the central pixel. This function may be used as sensor effectiveness function to model the effectiveness of the camera.

Alternatively, a function based on MTF can also be used. As we see from Figure 1, both metrics (MTF and QE) provide a similar model for effectiveness of a camera where the effectiveness of the camera is maximal at the centre and reduces with increase in distance from the central pixel.

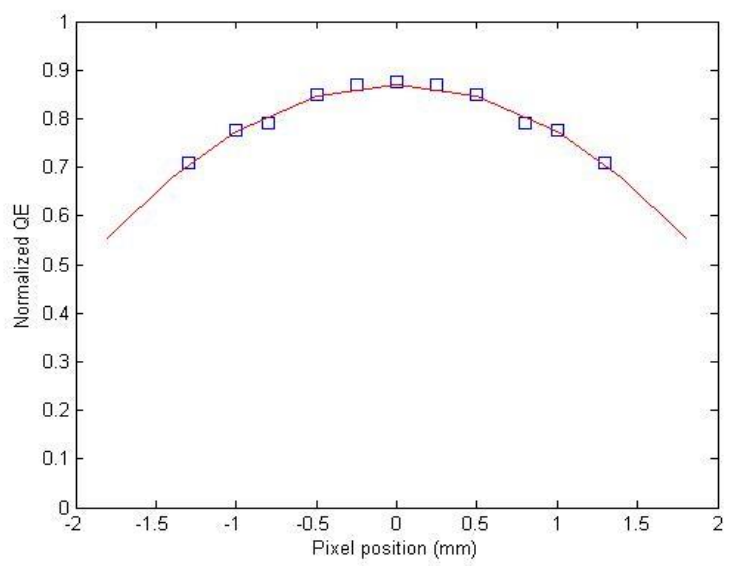

Fig. 1: QE v/s pixel position for 6m pixel size CMOS sensor. Adapted from [11].

\section{Optimal deployment of UAVs}

In this section we formulate the problem of optimal deployment of UAVs to maximize the search effectiveness of the cameras. We assume Eqn. (3) to model the effectiveness of the camera as search sensor. This model is similar to that of the sensor effectiveness function used in [4], in terms of being maximum at the center and monotonically decreasing away from the center. Hence, we formulate the optimal deployment problem based on that provided in [4], [12], [13].

We consider a problem of $\mathrm{N}$ UAVs carrying downward facing camera performing search operation in a search space $Q \in R^{2}$ The lack of information about presence (or absence) of the targets is modeled as an uncertainty density distribution $\phi: Q \rightarrow[0,1]$. The configuration of agents at any given time $\mathrm{t}$ is $P(t)=\left(p_{1}(t), p_{2}(t), \ldots . ., p_{N}(t) \in Q^{2}\right.$, with $p_{i}=p_{j}$, whenever $i \neq j$, where $p_{i}(t)$, is the position of the $\mathrm{i}$-th agent at time $\mathrm{t}$. After getting deployed in $\mathrm{Q}$, the UAVs collect information about the presence of targets of interest using onboard cameras, that is perform "search", thereby reducing the uncertainty density distribution over Q as,

$\phi_{-} \operatorname{nen}(q)=\phi_{\text {init }}(q) \min _{i}\left\{\beta\left(\left\|p_{i}-q\right\|\right)\right\}$

where, $\phi_{\text {init }}(q)$ is the initial uncertainty density (that is, before performing search); $\phi_{\text {new }}(q)$ is the uncertainty density after performing search; $\beta: \Re \rightarrow(0,1)=1-f$ is a strictly increasing function of the Euclidean distance from the center of UAV/camera as $f(\cdot)$ is assumed to be strictly decreasing function of distance from the central pixel) acting as a factor of reduction in uncertainty by the sensors. At a given $\mathrm{q} \in \mathrm{Q}$, we let only the UAV with the smallest $\beta\left(\left\|p_{i}-q\right\|\right)$, that is, the UAV which can reduce the uncertainty by the largest amount performs search. If all the agents search within their respective Voronoi cells, computed based on $\mathrm{P}$, then evaluating $\left.\min _{i}\left\{\beta\left\|p_{i}-q\right\|\right)\right\}$ in (4) is equivalent to evaluating $\left.\beta\left\|p_{i}-q\right\|\right)$, with $p_{i} \in V_{i}$, the Voronoi cell corresponding to the i-th agent.

\subsection{Objective function}

Now we may formulate an objective function which when maximized maximizes the reduction in uncertainty density, which 
is equivalent of increase in information (about presence of targets of interest) gain. Consider,

$$
\begin{aligned}
& H=\int_{Q} \Delta \phi(q) d Q \\
& H=\int_{Q}\left(\phi_{\text {init }}(q)-\phi_{\text {new }}(q)\right) d Q \\
& H=\sum_{i}^{N} \int_{V_{i}} \phi_{\text {init }}(q)\left(1-\beta\left(\left\|p_{i}-q\right\|\right)\right) d Q \\
& \left.H=\sum_{i}^{N} \int_{V_{i}} \phi_{\text {init }}(q) f\left(\left\|p_{i}-q\right\|\right)\right) d Q
\end{aligned}
$$

The gradient of the objective function $\mathscr{H}$ with respect to $p_{i}$ is given by,

$$
\frac{\partial H_{n}}{\partial p_{i}}=\int_{v_{i}} \phi_{i n i t}(q) \frac{\partial}{\partial\left(p_{i}\right)}\left(1-\beta\left(r_{i}\right)\right) d Q
$$

Where, $r_{i}=\left\|p_{i}-q\right\|$. The gradient given by (6) is spatially distributed over the Delaunay graph GD, where two agents $\mathrm{i}$ and $\mathrm{j}$ are considered neighbors if and only if $V_{i} \cap V_{j} \neq \phi$.

This im-

plies that each UAV can compute the gradient (6) on its own, thus not requiring a central computer for the same. The gradient (6) can be rewritten as,

$$
\begin{aligned}
& \frac{\partial H_{n}}{\partial p_{i}}=2 \int_{v_{i}} \phi(q) \frac{\partial f}{\partial\left(r_{i}^{2}\right)}\left(p_{i}-q\right) d Q \\
& \frac{\partial H_{n}}{\partial p_{i}}=-\int_{v_{i}} \phi^{\prime}\left(p_{i}-q\right) d Q=-\tilde{M}_{v_{i}}\left(p_{i}-\tilde{C}_{v_{i}}\right)
\end{aligned}
$$

Where, q, which can be interpreted as the density perceived by the sensor. As $f$ is a strictly decreasing function, $\tilde{\phi}(q)$ is always non-negative. Here $\tilde{M}_{v_{i}}$ and $\tilde{C}_{v_{i}}$ can be interpreted as the mass and centroid of the cell $V_{i}$ with $\tilde{\phi}$ as density. Thus, the critical points are, $p_{i}=\tilde{C}_{v_{i}}$ and such a configuration $\mathrm{P}$ of agents is called a centroidal Voronoi configuration. Note that $\tilde{C}_{v_{i}}$ depends on P. Further, for the sensor effectiveness function given in Eqn. (3), $\frac{\partial f}{\partial\left(r_{i}^{2}\right)}=-0.0714$.

That is, $\tilde{\phi}=0.1428$.Thus, $\tilde{C}_{v_{i}}=C_{v_{i}}$ where $C_{v_{i}}$ is the centroid of $V_{i}$ with $\phi$ as density. In Equation (3), modeling camera effectiveness, $r_{p i x}$ is the distance of a current pixel of interest from the center of image. However, in reality, we are interested in $r_{i}=\left\|p_{i}-q\right\|$, where $q$ is located in $Q$ rather than in the image frame. For each $q \in Q$, there is a corresponding pixel in the image frame.

A transformation between the $F O V_{i} \subset Q$, the field of view of the camera mounted on the i-th UAV, and its image frame needs to be established. This is illustrated in Figure 2.

Note further that, $p_{i}$ is assumed to be in $Q$. However, in reality, $Q$ is typically is a portion of the ground, while the UAV flies above the ground.

Thus, for the practical purposes, we use $p_{i}$ to be the point on ground (assumed to be flat for simplicity) directly below the UAV. Thus, for the practical purposes, we use $p_{i}$ to be the point on ground (assumed to be flat for simplicity) directly below the UAV. That is,

$$
p_{i}=\left(\begin{array}{c}
p_{x i} \\
p_{y i} \\
0
\end{array}\right)
$$

Where the location of the i-th UAV (strictly speaking the center of the image sensor of the camera carried by it is $\left(p_{x i}, p_{y i}, p_{z i}\right)^{T}$.

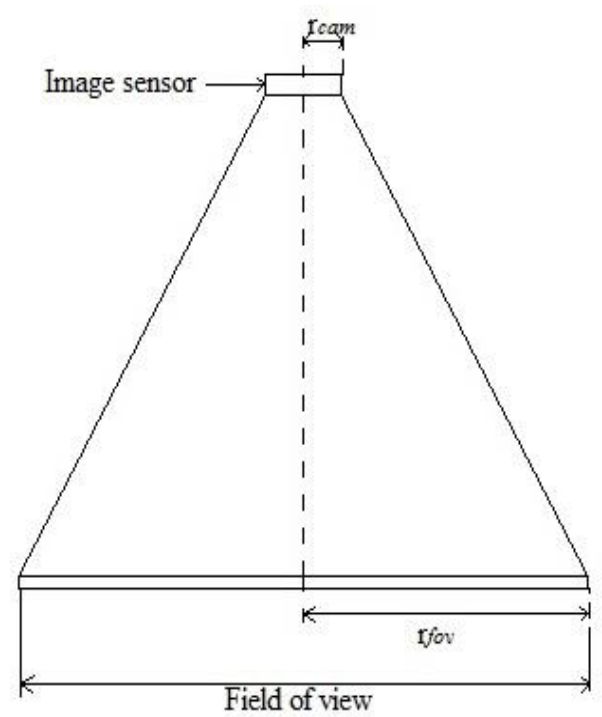

Fig. 2: Field of view to image frame transformation.

Now we have, $r_{i}=\left\|p_{i}-q\right\|=k r_{\text {pix }}$

Where the magnification factor,

$k=\frac{r_{f o v}}{r_{p i x}}$

Here, $r_{f o v}=\left\|p_{i}-q\right\|$, is the distance between the point on Q directly below the camera and the point of interest $q$.

\subsection{Control law}

Now consider a simple proportional control law [4],

$u_{i}(t)=-k_{\text {prop }}\left(p_{i}(t)-\left(\tilde{C}_{v_{i}}(t)\right)\right.$

Which makes the UAV move toward the corresponding centroid. In this work, we use a discretized version of this control law, where the UAV is given a motion toward the corresponding Voronoi cell centroid. In [4], authors have shown that for an agent having a simple first order dynamics (assumed to be a point mass), the control law (10), make the agents move asymptotically to the corresponding centroid. Note that the Voronoi cell itself is function of $\mathrm{P}$, the configuration of $\mathrm{N}$ UAVS, and hence as each UAV moves toward the computed centroid, the Voronoi cells change and hence the centroids keep shifting. Thus each UAV should chase a moving centroid. The strategy followed by each UAV is shown in Algorithm 1.

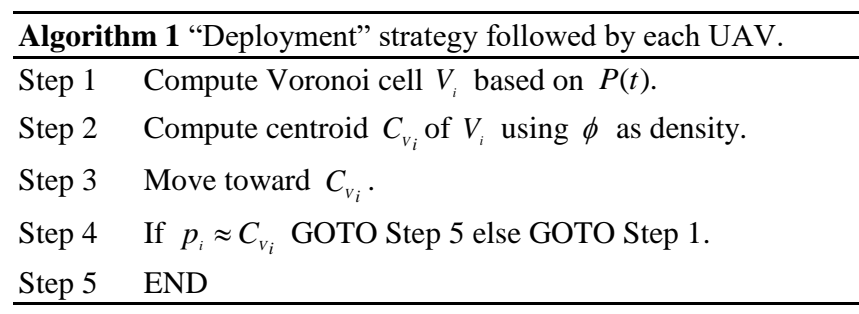

Once the optimal deployment is achieved, cameras perform search reducing the uncertainty density. The focus of this work being 
incorporating a realistic camera model suitable for a multi-UAV search problem, and using it to formulate an optimal deployment problem, we do not discuss the search process here. Also, we still use point mass models for the UAVs for the same reason. Simulation using realistic dynamic models of the UAVs (such as quadcopters) and incorporating search is part of ongoing work.

\section{Results and Discussions}

In this section we present the simulation results to demonstrate the proposed optimal deployment of multiple UAVs carrying downward facing cameras for a multi-UAV search application, using a realistic model for search effectiveness of the cameras. Initially al the UAVs are located at bottom left corner of the search space. As each UAV moves toward the centroid of the corresponding Voronoi cell, note that the UAVs are getting more uniformly distributed. We have simulated in MATLAB for the optimal deployment

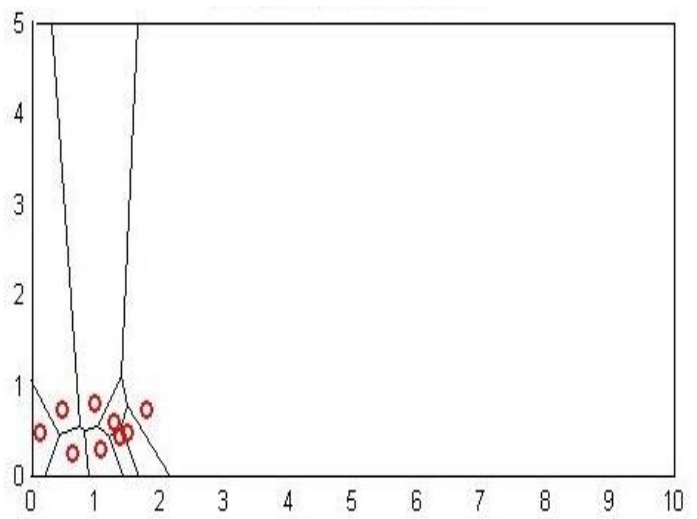

(a)

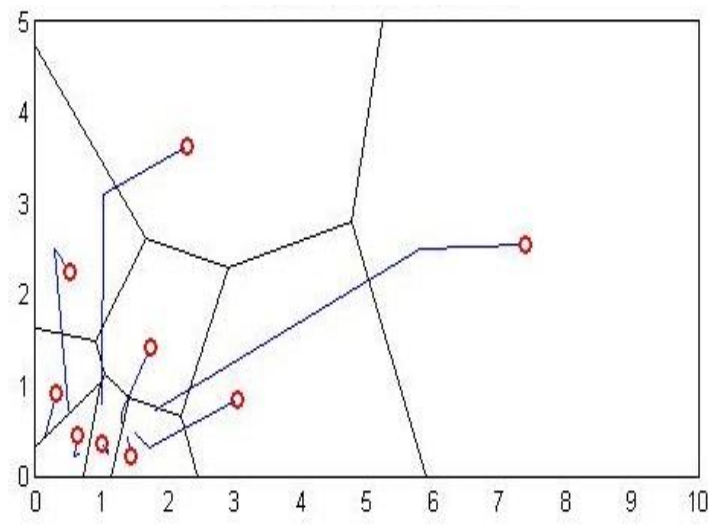

(c)

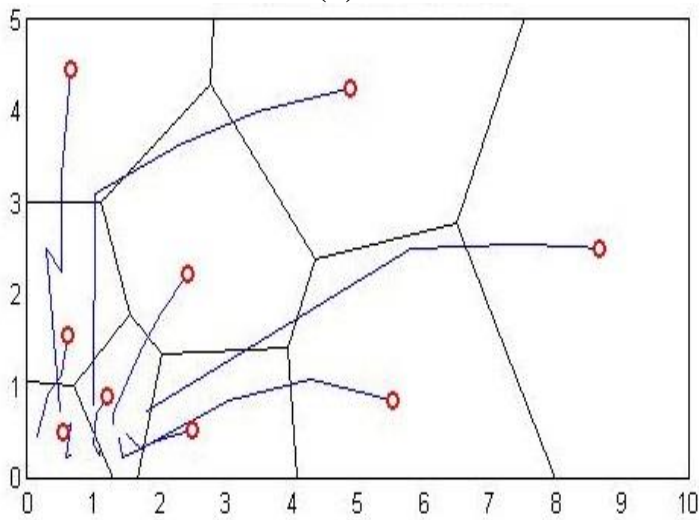

(e) of 9 UAVs carrying downward facing cameras. The deployment process is shown in Figures 3(a) - (h). Figures 3(a) shows the initial configuration of the UAVs along with the corresponding Voronoi cells. Figures 3(b) - (h) show the intermediate configuration at the end of each iteration, and finally the deployment of UAVs into the centroidal Voronoi configuration, that is, the optimal configuration, is shown in Figure 3 (h).

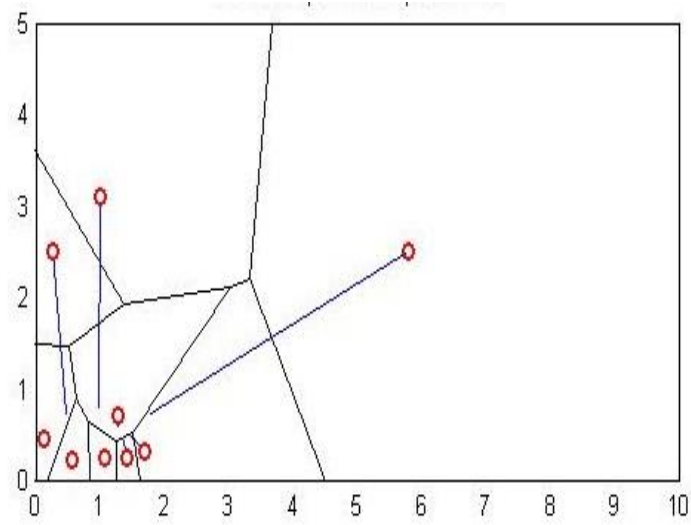

(b)

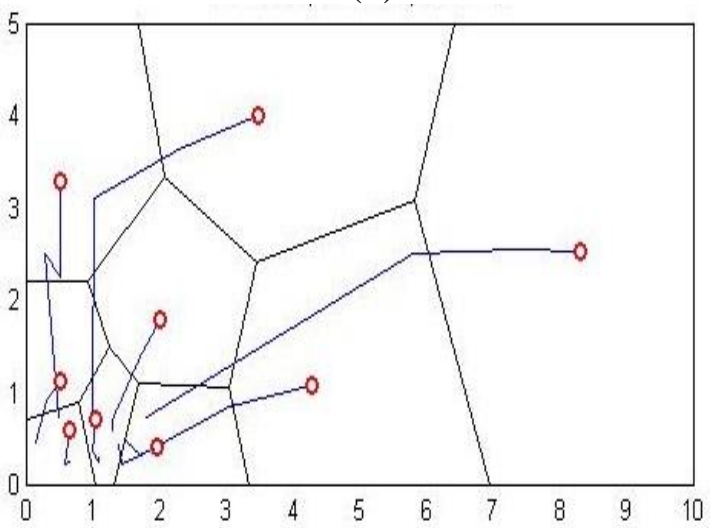

(d)

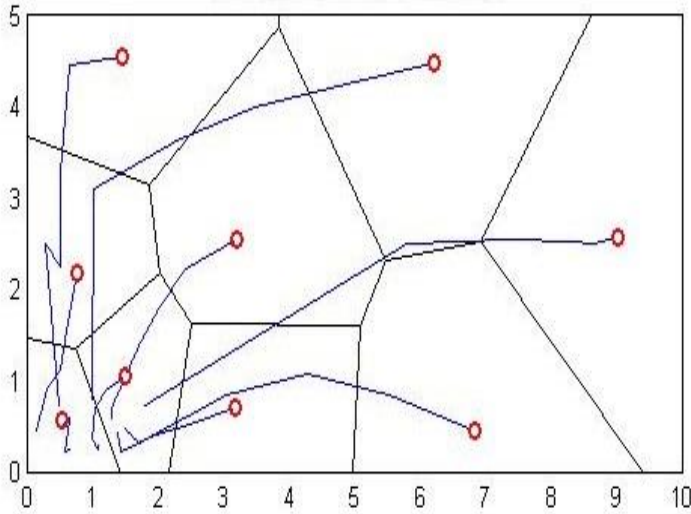

(f) 


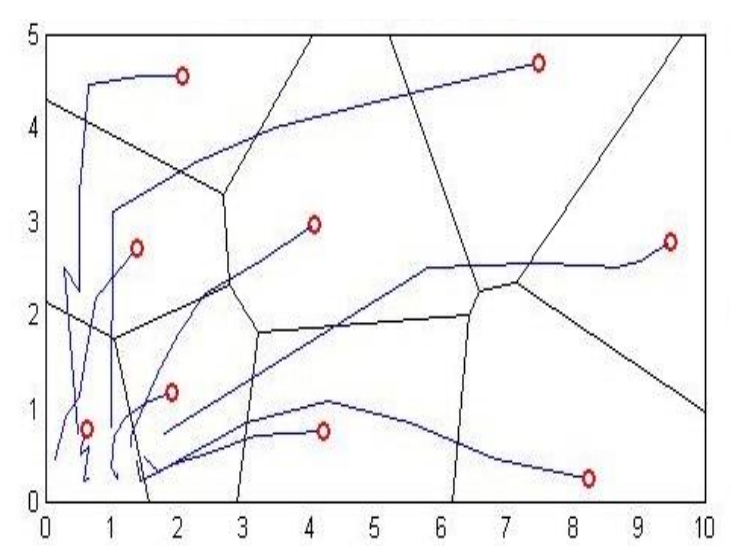

(g)

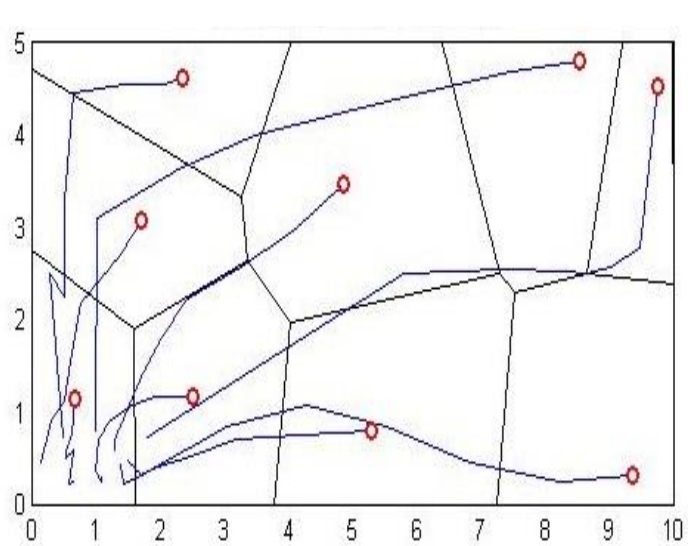

(h)

Fig. 3. Snapshots of the process of deployment of UAVs from (a) an initial configuration into (e) a centroidal Voronoi configuration. The centroid of each Voronoi cell is marked with 'o' and the position of the robot is marked with '+'.

\section{Conclusion}

We formulated a multi-UAV optimal deployment problem for a multi-UAV search application using downward facing cameras mounted on each of the UAVs as search sensors. We presented a discussion on modeling sensor effectiveness function of a camera with the pixel location, and equivalently, with the distance away from the center of the camera/UAV. We used a sensor effectiveness model based on the Quantum Efficiency and used to it formulate an objective function, which when maximized, maximizes the search effectiveness in terms of information gain or uncertainty reduction. We demonstrated the proposed optimal deployment strategy using a simple simulation in MATLAB, and the results showed that the proposed deployment strategy succeeded in achieving an uniform deployment of UAVs (and hence the cameras) using the centroidal Voronoi configuration. The current work focussed on obtaining a suitable camera effectiveness model for the purpose of optimal deployment of UAVs for a search application. Though we used a sensor effectiveness model based on the concept of the Quantum efficiency, there are several alternatives such as MTF. Further, the model also depends on the application in terms typical altitude of UAVs, the scenario (high contrast or low contrast targets), resolution required to successfully detect the targets with minimal false detection, and finally the properties of the cameras used. The sensor effectiveness function may be obtained experimentally. Such an experimental investigation is part of ongoing work. Further, we have restricted to simulation of point masses in place of realistic dynamics of the UAVs for simplicity here. Carrying out simulation incorporating dynamics of UAVs (quadcopters) and experimental investigation using physical quadcopters are also part of ongoing/future work.

\section{References}

[1] Adams, M. Stuart and J. Friedland Carol, "A survey of unmanned aerial vehicle (UAV) usage for imagery collection in disaster research and management", Proceedings of 9th International Workshop on Remote Sensing for Disaster Response, 2011.

[2] G.D. Boreman, "Modulation Transfer Function in Optical and Electro-Optical Systems", SPIE Press, Bellingham, WA, 2001.

[3] Rodríguez-Canosa, G.R.; Thomas, S.; del Cerro, J.; Barrientos, A.; MacDonald, B. "A Real-Time Method to Detect and Track Moving Objects (DATMO) from Unmanned Aerial Vehicles (UAVs) Using a Single Camera", Remote Sens. 2012, 4, 10901111.

[4] Guruprasad K.R. and Ghose D., "Automated multi-agent search using centroidal voronoi configuration", IEEE Tansactions on
Automation Science and Engineering, vol. 8, issue 2, April 2011, pp. 420 - 423.

[5] Sujit P.B. and Ghose D., "Search using multiple UAVs with flight time constraints", IEEE Trans. on Aerospace and Electronic Systems, vol. 40, no. 2, April 2004, pp. 491-509.

[6] Zhang Minqiang, Song Jianmei, Huang Lan, and Zhang Chunyan, "Distributed cooperative search with collision avoidance for a team of unmanned aerial vehicles using gradient optimization", Journal of Aerospace Engineering, vol 30, issue 1, january 2017.

[7] Schwager Mac, Julian Brian J., Angermann Michael, and Rus Daniela, "Eyes in the Sky: Decentralized Control for the Deployment of Robotic Camera Networks", Proceedings of the IEEE, vol. 99, no. 9, September 2011, pp. 1541-1561.

[8] Cortes J., Martinez S., Karata T., and Bullo F., Coverage control for mobile sensing networks, IEEE Transactions on Robotics and Automation, vol. 20, no. 2, 2004, pp. 243-255.

[9] Warren J. Smith, Modern Optical Engineering, Third Edition, McGraw-Hill Professional, 2000.

[10] V. Sukumar, Herbert L. Hess, Kenneth V. Noren, Gregory Donohoe, and Suat Ay, "Imaging system MTF-modeling with modulation functions", in Proceedings of 34th Annual IEEE Conference of Industrial Electronics (IECON), 2008.

[11] Ting Chen, "Digital camera system simulator and applications", Ph.D. thesis, Stanford University, 2003.

[12] K.R. Guruprasad, "Multiagent search strategy based on centroidal Voronoi configuration", 2010 IEEE International Conference on Robotics and Automation, 05/2011.

[13] http://info.adimec.com/blogposts/ccd-vs.-cmos-sensitivity-inlow-light-improvements-with-industrial-cmos-image-sensorsand-cameras, CCD Vs. CMOS, Sensitivity in low light improvements with industrial CMOS image sensors and cameras posted by Gretchen Alper, March 12, 2015. 